Check for updates

Cite this: RSC Adv., 2018, 8, 35280

\title{
The glycine-rich flexible region in SSB is crucial for PriA stimulation
}

\begin{abstract}
Yen-Hua Huang ${ }^{a}$ and Cheng-Yang Huang (D) *ab
Single-stranded DNA-binding protein (SSB) is essential for all DNA-dependent cellular processes. The mechanism through which PriA helicase, an initiator protein in the DNA replication restart process, is stimulated by SSB in Escherichia coli (EC) has been established. Nevertheless, whether or not PriA stimulated by SSB is conserved among Gram-negative bacteria remains unclear, and the SSB specificity for the stimulation effect on PriA is unknown. In this study, three similar SSBs from Klebsiella pneumoniae (KpSSB), Salmonella enterica (StSSB), and Pseudomonas aeruginosa (PaSSB) were used to analyze the stimulation effect. Two chimeric proteins, namely, KpSSBn-PaSSBc and KpSSBn-StSSBc, were also used. KpSSB, StSSB, and KpSSBn-StSSBc can stimulate KpPriA activity, but PaSSB and KpSSBnPaSSBc cannot. The crystal structure of PaSSB solved at $2.04 \AA$ resolution (PDB entry 5YUO) reveals the classic OB fold structure, similar to that of EcSSB. Comparison of SSBs through sequence analysis showed that the typical glycine-rich flexible region in PaSSB contains very few glycine residues. Through analyses of protein chimeragenesis, structure-sequence, and ATPase stimulation effects, we concluded that the inherent difference in the glycine-rich flexible region among SSB species is a determinant of PriA stimulation. Further research can directly focus on determining the type of glycine-rich hinge that can stimulate PriA and the reason why bacterial SSBs need to evolve different C-terminal domains during evolution.
\end{abstract}

Received 1st September 2018

Accepted 9th October 2018

DOI: $10.1039 / \mathrm{c} 8 \mathrm{ra07306f}$

rsc.li/rsc-advances chromosome, other proteins are also involved in this process, such as PriB, PriC, DnaT, and DnaC. The E. coli model shows at least two DnaB helicase-recruiting pathways: (i) the PriA-PriBDnaT-DnaC-dependent reactions are the most effective on fork structures with no gaps in the leading strand, whereas (ii) the PriC-DnaC-dependent system preferentially uses fork structures with large gaps in the leading strand. ${ }^{9}$ Due to PriC-DnaT interactions, a putative link has been proposed between these two independent replication restart pathways. ${ }^{10-15}$ Klebsiella pneumoniae (Kp) also have these primosomal proteins to restart the DNA replication. However, we found that Pseudomonas aeruginosa $(\mathrm{Pa})$ does not contain any recognizable homolog of priB, priC, dnaT, and dnaC in its genome. Only PriA and SSB are found in $P$. aeruginosa. Thus, it is impossible to follow the replication restart strategy of $E$. coli and $K$. pneumoniae for $P$. aeruginosa. How DNA replication can restart in $P$. aeruginosa is still unknown (Fig. 1). Because of lacking experimental evidences, nothing is known whether or not PaSSB can stimulate PriA helicase.

$P$. aeruginosa is a major cause of life-threatening infections in burn patients worldwide and has a remarkable capacity to develop resistance to multiple classes of antimicrobial agents. ${ }^{16}$ The increased prevalence of $\beta$-lactamases in $P$. aeruginosa has begun to reduce the clinical efficacy of $\beta$-lactams against the most common opportunistic pathogens. To date, over $800 \beta$ lactamases have been identified, of which at least $120 \beta$ -

${ }^{a}$ School of Biomedical Sciences, Chung Shan Medical University, No. 110, Sec. 1, Chien-Kuo N. Rd., Taichung City, Taiwan

${ }^{b}$ Department of Medical Research, Chung Shan Medical University Hospital, No. 110 Sec. 1, Chien-Kuo N. Rd., Taichung City, Taiwan.E-mail: cyhuang@csmu.edu.tw; Tel: +886-4-24730022 ext. 11472 

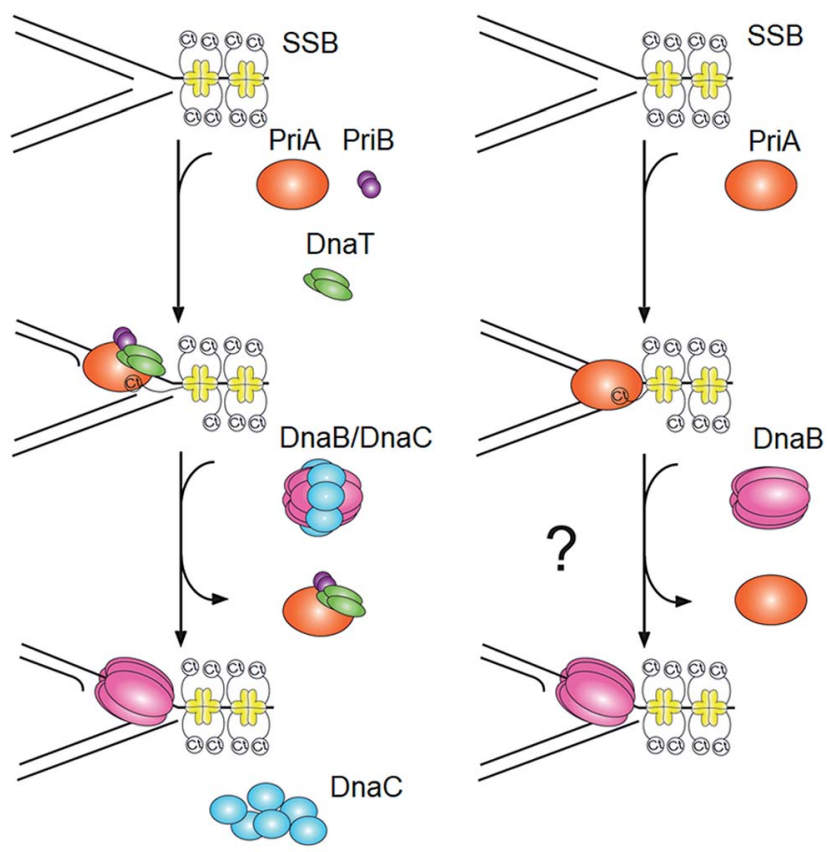

Replication restart
E. coli/ K. pneumoniae

Replication restart

$P$. aeruginosa

Fig. 1 The PriA-directed DNA replication restart pathway. In E. coil and K. pneumoniae, the PriA-PriB-DnaT-DnaC-dependent reactions are important for recalling the DnaB helicase back onto the chromosome. However, $P$. aeruginosa does not contain any recognizable homolog of priB, dnaT, and dnaC in its genome. $P$. aeruginosa also does not have PriC protein, an initiator in another DNA replication restart pathway. For restart system, only PriA and SSB are found in $P$. aeruginosa.

lactamases have been detected in $P$. aeruginosa. ${ }^{17}$ Recently, some SSB inhibitors as broad-spectrum antibacterial agents targeting $P$. aeruginosa and other pathogens have been discovered. ${ }^{18,19}$ Thus, PaSSB structure may be needed as a basis for rational drug design to target $P$. aeruginosa.

Table 1 Primers used for construction of plasmids ${ }^{a}$

Oligonucleotide Primer

$\begin{array}{ll}\text { KpPriA-EcoRI-N } & \text { ATATAGAATTC ATGTCCGTCGCCCACG } \\ \text { KpPriA-HindIII-C } & \text { TGGTGAAGCTT TCCTTCAATCGGATCG } \\ \text { PaPriA-EcoRI-N } & \text { TACGGGATTC ATGTCAGACCTGATCC } \\ \text { PaPriA-HindIII-C } & \text { TTGCTAAGCTT GAACAGGTCGATCGGA } \\ \text { PaSSB(W40A)-N } & \text { ACCCTCGCCACCAGCGAGAGCGC GAAGGACAAG } \\ \text { PaSSB(W40A)-C } & \text { CGGTCTGCTTGTCCTTCGC GCTCTCGCTGGTGG } \\ \text { PaSSB(W54A)-N } & \text { CCAGCAACAGGAGCGCACCGAAGC GCACCGCGT } \\ \text { PaSSB(W54A)-C } & \text { GAAGAACACCACGCGGTGCGC TTCGGTGCGCTC } \\ \text { PaSSB(F60A)-N } & \text { GAATGGCACCGCGTGGTGTCGC CGGCCGCCTG } \\ \text { PaSSB(F60A)-C } & \text { GCGATCTCCGCCAGGCGGCCGGC GAACACCACG } \\ \text { PaSSB(W88A)-N } & \text { GGCAGCCTGCGCACCCGCAAGGCGCAGGGCAGG } \\ \text { PaSSB(W88A)-C } & \text { CGATCCTGACCGTCCTGCCCTGCGCCTTGCGGG }\end{array}$

${ }^{a}$ These plasmids were verified by DNA sequencing. Underlined nucleotides indicate the designated site for the restriction site or the mutation site.
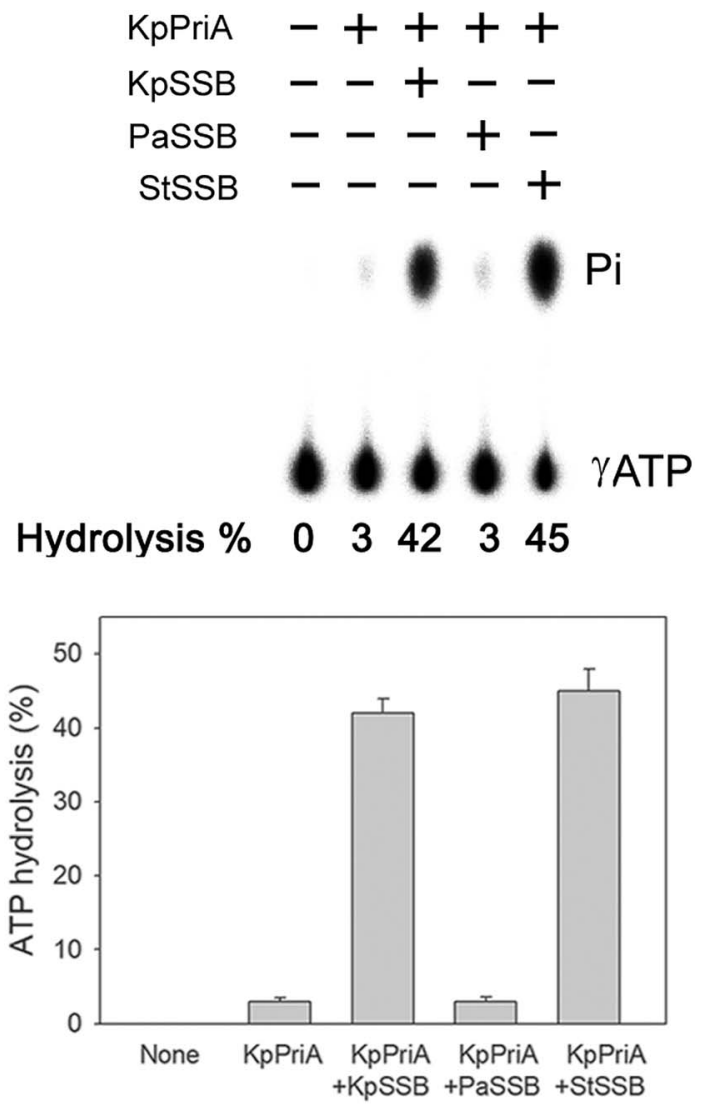

Fig. 2 The ATPase activity of KpPriA did not change when acting with PaSSB. KpPriA ATPase assay was performed with $0.4 \mathrm{mM}\left[\gamma^{-}{ }^{32} \mathrm{P}\right]$ ATP and $0.0625 \mu \mathrm{M}$ KpPriA in reaction buffer containing $40 \mathrm{mM}$ Tris $(\mathrm{pH}$ 8.0), $10 \mathrm{mM} \mathrm{NaCl}, 2 \mathrm{mM}$ DTT, $2.5 \mathrm{mM} \mathrm{MgCl}_{2}$, and $0.1 \mu \mathrm{M}$ PS4/PS3dT30 DNA substrate. To study the effect, KpSSB $(10 \mu M), \operatorname{StSSB}(10 \mu M)$, or PaSSB $(10 \mu \mathrm{M})$ was added into the assay solution. Aliquots $(5 \mu \mathrm{L})$ were taken and spotted onto a polyethyleneimine cellulose thin-layer chromatography plate, which was subsequently developed in $0.5 \mathrm{M}$ formic acid and $0.25 \mathrm{M} \mathrm{LiCl}$ for $30 \mathrm{~m}$. Reaction products were visualized by autoradiography and quantified with a phosphorimager.

KpSSB, ${ }^{20}$ Salmonella enterica serovar Typhimurium LT2 SSB (StSSB), ${ }^{21}$ and $\mathrm{PaSSB}^{22}$ are similar proteins with identical $\mathrm{N}$ terminal ssDNA-binding domains. ${ }^{23}$ However, the C-terminal domain of PaSSB (aa 116-165) contains only one Gly residue, which is significantly less than that of KpSSB ( 11 Gly residues) and StSSB (12 Gly residues). Considering that Gly (and Pro) is an important component of the flexible region in protein, we should assess whether the glycine-rich hinge that is not conserved among SSBs is involved in the stimulation of the PriA helicase.

The length of the glycine-rich hinge in EcSSB is found to affect EcPriA activity. ${ }^{24}$ Whether different glycine-rich hinges of SSB can affect PriA activity is still unknown. In this study, we found that KpSSB and StSSB, but not PaSSB, can stimulate KpPriA activity. PaSSB also could not enhance PaPriA activity. The crystal structure of the N-terminal domain of PaSSB solved at $2.04 \AA$ resolution (PDB entry 5YUO) revealed the classic OB fold structure, similar to that of EcSSB. We swapped the Cterminal domains of PaSSB and StSSB into that of KpSSB 
through protein chimeragenesis. Similar to PaSSB, the chimeric protein KpSSBn-PaSSBc showed no stimulation effect on KpPriA. Basing on the analyses of ATPase stimulation effects, structure-sequence, and protein chimeragenesis, we concluded that not only the C-terminal acidic tail but also the flexible glycine-rich hinge in SSB are important for stimulation of PriA activity. Further research can directly find the reason why bacterial SSBs need to have different C-terminal domains during evolution.

\section{Experimental}

Construction of plasmids for PaPriA, KpPriA, KpSSB, StSSB, PaSSB, KpSSBn-StSSBc, and KpSSBn-PaSSBc expression

Construction of the KpSSB, StSSB, PaSSB, KpSSBn-StSSBc, and KpSSBn-PaSSBc expression plasmids has been reported. ${ }^{23}$ $K P N 04230$, the gene encoding a putative KpPriA, was amplified through PCR by using the genomic DNA of $K$. pneumoniae subsp. pneumonia MGH 78578 as template. PA5050, the gene encoding a putative PaPriA, was amplified through PCR by using the genomic DNA of $P$. aeruginosa PAO1. The primers used for the construction of the pET21-KpPriA and pET21PaPriA plasmids are summarized in Table 1.

\section{Protein expression and purification}

Purification of the recombinant KpSSB, StSSB, PaSSB, $\mathrm{KpSSBn}-\mathrm{StSSBc}$, and KpSSBn-PaSSBc protein has been reported..$^{23}$ pET21b-KpSSBnStSSBc and pET21b-KpSSBnPaSSBc will express KpSSB1-91 fused StSSB92-176 and PaSSB91-165, respectively. The recombinant KpPriA and PaPriA proteins were expressed and purified using the protocol described previously for PriB. ${ }^{25-27}$ Briefly, E. coli BL21(DE3) cells were transformed with the expression vector and overexpression of the expression plasmids was induced by incubating with $1 \mathrm{mM}$ isopropyl thiogalactopyranoside. The protein was purified from the soluble supernatant by $\mathrm{Ni}^{2+}$-affinity chromatography (HiTrap HP; GE Healthcare Bio-Sciences), eluted with Buffer A (20 mM Tris- $\mathrm{HCl}, 250 \mathrm{mM}$ imidazole, and $0.5 \mathrm{M}$ $\mathrm{NaCl}, \mathrm{pH}$ 7.9), and dialyzed against a dialysis buffer $(20 \mathrm{mM}$ HEPES and $100 \mathrm{mM} \mathrm{NaCl}, \mathrm{pH}$ 7.0; Buffer B). Protein purity remained at $>97 \%$ as determined by SDS-PAGE (MiniPROTEAN Tetra System; Bio-Rad, CA, USA).

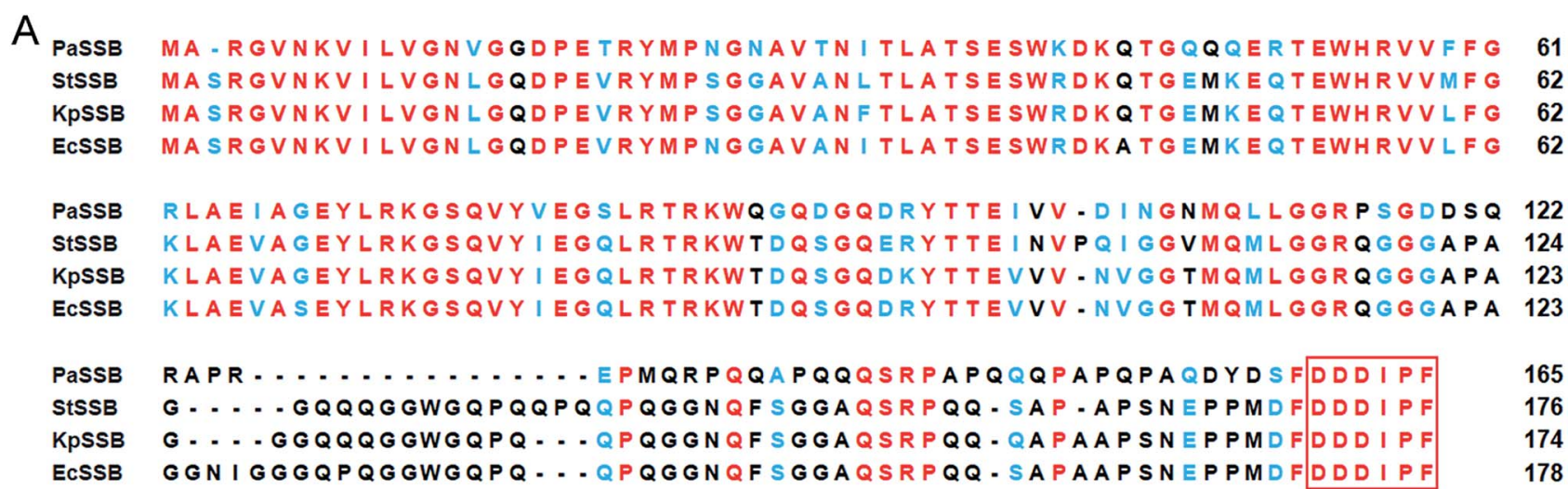

B

1

M A R G V N K V I L

51

R T E W H V V F F

101

I V V I N G N Q

151

Q P A D Y D F D
11

V G N V G D P T

61

G R L A E I A G E Y

111

L I G G R P S G D D

161

D D I P F
21

R Y M P N G N A T T

71

L R K G S Q V Y V E

121

S Q R A P R P M Q

31

N I T LA T S E S W

81

G S L R T R K W Q G

131

R P Q Q A P Q Q S

41

K D K Q T G Q Q Q E

91

Q D G Q D R Y T T E

141

R P A P Q Q Q P A P

\begin{tabular}{r|rlllll|l|l}
1 & 2 & 3 & 4 & 5 & 6 & 7 & 8 & 9 \\
Variable & \multicolumn{4}{c|}{ Average } & \multicolumn{3}{c}{ Conserved }
\end{tabular}

Fig. 3 (A) Multiple amino acid sequence alignment of SSB proteins. Sequence alignment of PaSSB, StSSB, KpSSB, and EcSSB was generated by CLUSTALW2. Identical amino acid residues are colored in red. Amino acid residues with similar properties judged by CLUSTALW2 are colored in light blue. The $\mathrm{C}$-terminal acidic tail involved in PriA binding is boxed. The amino acid residues in the $\mathrm{C}$-terminal long glycine-rich hinge of PaSSB (aa 116-158) are variable. (B) An alignment consensus of 150 sequenced bacterial SSB homologs by ConSurf reveals the degree of variability at each position along the primary sequence. Highly variable amino acid residues are colored teal, whereas highly conserved amino acid residues are burgundy. A consensus sequence was established by determining the most commonly found amino acid residue at each position relative to the primary sequence of PaSSB. 
Table 2 Data collection and refinement statistics

\begin{tabular}{|c|c|}
\hline \multicolumn{2}{|l|}{ Data collection } \\
\hline Crystal & PaSSB \\
\hline Wavelength $(\AA)$ & 0.975 \\
\hline Resolution $(\AA)$ & $30-2.04$ \\
\hline Space group & $C_{1} 2_{1}$ \\
\hline Cell dimension $(\AA)$ & $\begin{array}{l}a=102.2 \alpha=90 \\
b=64.1 \beta=113.0 \\
c=97.1 \gamma=90\end{array}$ \\
\hline Completeness (\%) & $98.2(89.9)^{a}$ \\
\hline$\langle I / \sigma I\rangle$ & $31.36(2.53)$ \\
\hline$R_{\text {sym }}$ or $R_{\text {merge }}(\%)$ & $0.038(0.465)^{b}$ \\
\hline Redundancy & $3.6(3.1)$ \\
\hline \multicolumn{2}{|l|}{ Refinement } \\
\hline Resolution $(\AA)$ & $26.49-2.04$ \\
\hline No. reflections & 36399 \\
\hline$R_{\text {work }} / R_{\text {free }}$ & $0.217 / 0.257$ \\
\hline \multicolumn{2}{|l|}{ No. atoms } \\
\hline Protein & 408 \\
\hline Water & 87 \\
\hline \multicolumn{2}{|l|}{ R.m.s deviation } \\
\hline Bond lengths $(\AA)$ & 0.007 \\
\hline Bond angles $\left({ }^{\circ}\right)$ & 0.844 \\
\hline \multicolumn{2}{|l|}{ Ramachandran plot } \\
\hline In preferred regions & $384(98.46 \%)$ \\
\hline In allowed regions & $6(1.54 \%)$ \\
\hline Outliers & $0(0 \%)$ \\
\hline PDB entry & $5 Y U O$ \\
\hline
\end{tabular}

\section{ATPase assay}

KpPriA ATPase assay using the protocol described previously for SaPriA ${ }^{28-30}$ was performed with $0.4 \mathrm{mM}\left[\gamma^{32} \mathrm{P}\right]$ ATP and $0.0625 \mu \mathrm{M}$ KpPriA in reaction buffer containing $40 \mathrm{mM}$ Tris (pH 8.0), $10 \mathrm{mM} \mathrm{NaCl}, 2 \mathrm{mM}$ DTT, $2.5 \mathrm{mM} \mathrm{MgCl}_{2}$, and $0.1 \mu \mathrm{M}$ PS4/PS3-dT30 DNA substrate. Aliquots $(5 \mu \mathrm{L})$ were taken and spotted onto a polyethyleneimine cellulose thin-layer chromatography plate, which was subsequently developed in $0.5 \mathrm{M}$ formic acid and $0.25 \mathrm{M} \mathrm{LiCl}$ for $30 \mathrm{~m}$. Reaction products were visualized by autoradiography and quantified with a phosphorimager.

\section{Crystallography}

Before crystallization, PaSSB was concentrated to $20 \mathrm{mg} \mathrm{mL}^{-1}$ in Buffer B. Crystals were grown at room temperature by hanging drop vapor diffusion in 14\% PEG 4000, $100 \mathrm{mM}$ HEPES, $50 \mathrm{mM}$ sodium acetate, $\mathrm{pH}$ 7.0. Data were collected using an ADSC Quantum-315r CCD area detector at SPXF beamline BL13C1 at NSRRC (Taiwan, ROC). All data integration and scaling were carried out using HKL-2000. ${ }^{31}$ There were four PaSSB monomers per asymmetric unit. The crystal structure of PaSSB was solved at $2.04 \AA$ resolution with the molecular replacement software Phaser-MR ${ }^{32}$ using EcSSB as model (PDB entry 1EYG). A model was built and refined with PHENIX ${ }^{33}$ and Coot. $^{34}$ The final structure was refined to an $R$-factor of 0.217 and an $R_{\text {free }}$ of 0.257 .
Atomic coordinates and related structure factors have been deposited in the PDB with accession code 5YUO.

\section{Preparation of dsDNA substrate}

The double-stranded DNA substrate (dsDNA) PS4/PS3-dT30 for ATPase assay was prepared at a $1: 1$ concentration ratio. PS4/ PS3-dT30 was formed in $20 \mathrm{mM}$ HEPES (pH 7.0) and $100 \mathrm{mM}$ $\mathrm{NaCl}$ by briefly heating at $95^{\circ} \mathrm{C}$ for $5 \mathrm{~min}$ and by slowly cooling to room temperature overnight.

\section{Electrophoretic mobility shift assay (EMSA)}

EMSA for PaSSB was conducted in accordance with a previously described protocol for SSB. ${ }^{35}$ In brief, dT50 was radiolabeled with $\left[\gamma^{32} \mathrm{P}\right]$ ATP $\left(6000 \mathrm{Ci} \mathrm{mmol}^{-1}\right.$; PerkinElmer Life Sciences, Waltham, MA) and T4 polynucleotide kinase (Promega, Madison, WI, USA). The protein $(0,0.02,0.039,0.078,0.1563,0.3125$, $0.625,1.25,2.5$ and $5.0 \mu \mathrm{M}$ ) was incubated for $30 \mathrm{~min}$ at $25^{\circ} \mathrm{C}$ with $1.7 \mathrm{nM}$ DNA substrates in a total volume of $10 \mu \mathrm{L}$ in $20 \mathrm{mM}$ Tris- $\mathrm{HCl}(\mathrm{pH} 8.0)$ and $100 \mathrm{mM} \mathrm{NaCl}$. Aliquots $(5 \mu \mathrm{L})$ were removed from each of the reaction solutions and added to $2 \mu \mathrm{L}$ of gel-loading solution $(0.25 \%$ bromophenol blue and $40 \%$ sucrose). The resulting samples were resolved on $8 \%$ native polyacrylamide gel at $4{ }^{\circ} \mathrm{C}$ in TBE buffer $(89 \mathrm{mM}$ Tris borate and $1 \mathrm{mM}$ EDTA) for $1 \mathrm{~h}$ at $100 \mathrm{~V}$ and visualized through phosphorimaging. A phosphor storage plate was scanned, and data regarding complex and free DNA bands were digitized for quantitative analysis. The ssDNA binding ability of the protein was estimated through linear interpolation from the concentration of the protein that bound $50 \%$ of the input DNA.

\section{Results and discussion}

\section{KpSSB and StSSB can stimulate KpPriA activity, but PaSSB cannot}

PriA is known as a poor helicase when acting alone in vitro. ${ }^{36}$ EcPriA activity can be stimulated by EcSSB ${ }^{6}$ However, the Grampositive bacterial SSBs, namely, Staphylococcus aureus $\mathrm{SsbA},{ }^{29}$ $\mathrm{SsbB}^{28}$ and $\mathrm{SsbC}^{19}$ cannot stimulate SaPriA activity. These results suggest the presence of different levels of SSB specificity for the stimulation effect of PriA. As shown in Fig. 2, KpPriA could hydrolyze ATP alone, and this ATPase activity was dramatically stimulated (14-fold) in the presence of KpSSB. KpPriA activity was also stimulated by StSSB (15-fold), but not PaSSB. We also found that PaSSB did not enhance the PaPriA activity (data not shown). Thus, inherent differences among the Gram-negative bacterial SSB species may result in different effects on PriA stimulation.

\section{Sequence analysis of PaSSB}

KpPriA activity was stimulated by KpSSB and StSSB, but not PaSSB (Fig. 2). For the first time, the inability of the Gramnegative bacterial SSB (i.e., PaSSB) to stimulate PriA was found. KpSSB, StSSB, and PaSSB shares strong sequence similarity with the N-terminal DNA-binding domain (aa 1-110) and the C-terminal acidic tail of EcSSB (Fig. 3A). Fig. 3B shows the alignment consensus of 150 sequenced bacterial SSB homologs 
A

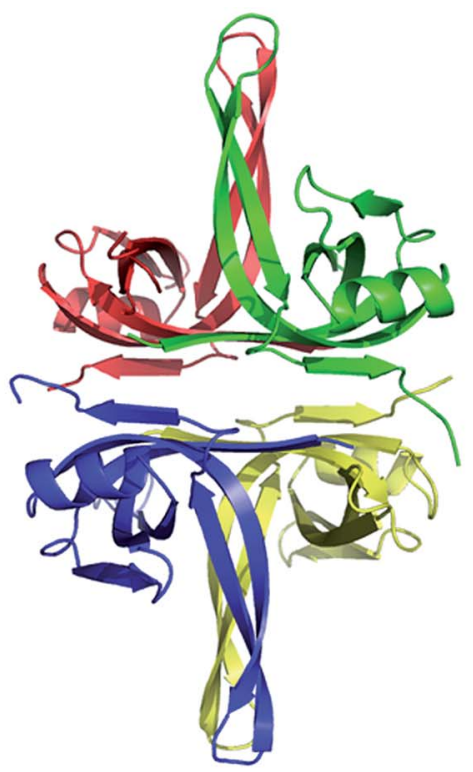

$\mathrm{C}$

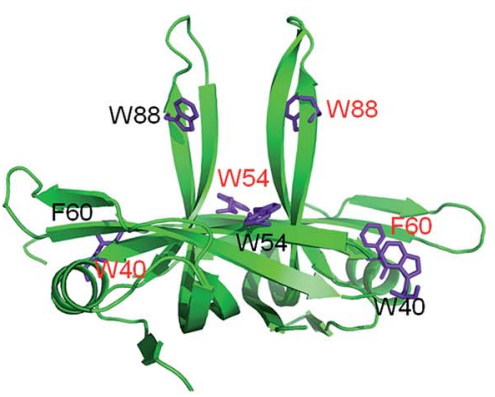

$\mathrm{D}$

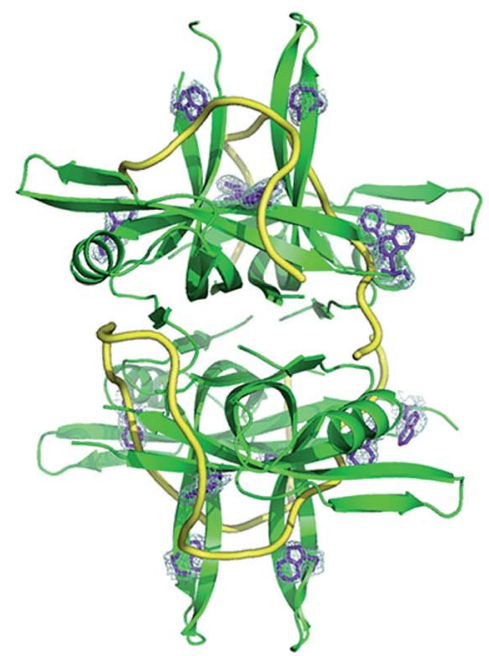

B
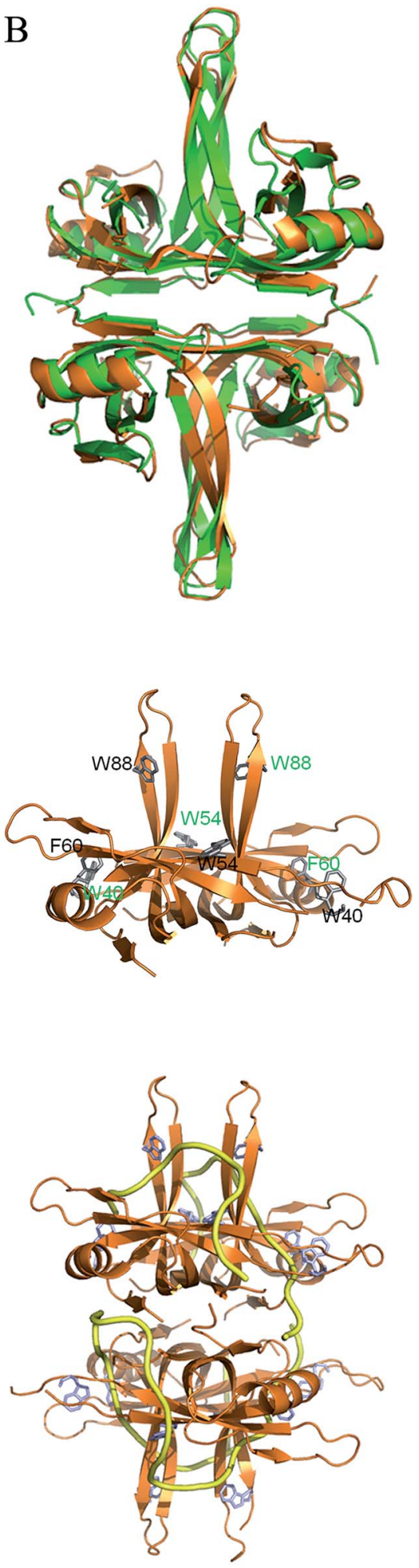

Fig. 4 (A) Crystal structure of PaSSB. Four monomers of PaSSB were found per asymmetric unit, and the core of the OB-fold possesses a $\beta$ barrel capped by an $\alpha$-helix. The entire C-terminal domain of PaSSB (aa 116-165) became disordered and disappeared. (B) Superposition of PaSSB and EcSSB. The N-terminal domains of PaSSB and EcSSB (PDB entry 1EYG; orange) are similar. (C) sSDNA-binding mode of PaSSB. In the EcSSB-ssDNA complex, four essential aromatic residues, including Trp40, Trp54, Phe60, and Trp88, participate in ssDNA binding via stacking interactions. The structurally corresponding residues in PaSSB are Trp40, Trp54, Phe60, and Trp88. (D) Based on the structural similarity between PaSSB and EcSSB (PDB entry 1EYG; orange), Trp40, Trp54, Phe60, and Trp88 in PaSSB may play a similar role in ssDNA binding as those in EcSSB. The ssDNA generated from the ssDNA-EcSSB complex is shown in gold in the PaSSB-ssDNA complex. The composite omit map (at 1.0 $\sigma$ ) showed the electron density of Trp40, Trp54, Phe60, and Trp88 in PaSSB. 


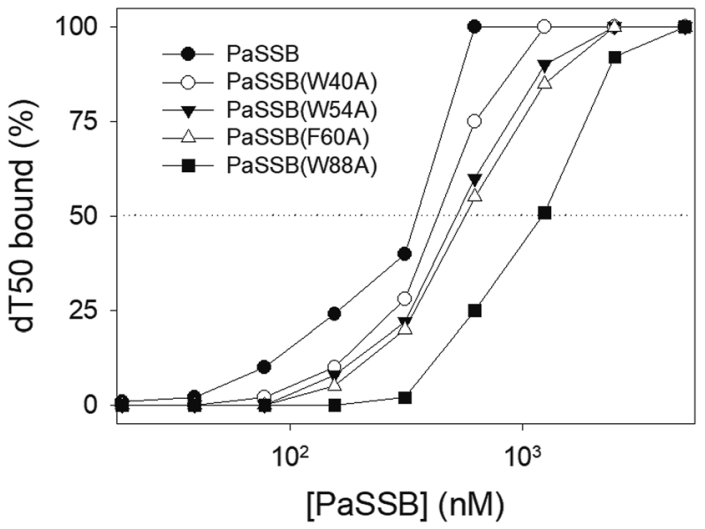

Fig. 5 Mutational analysis of PaSSB for ssDNA binding. PaSSB mutant protein (W40A, W54A, F60A, and W88A) was incubated at $25^{\circ} \mathrm{C}$ for $30 \mathrm{~min}$ with dT50 in a total volume of $10 \mathrm{~mL}$ in $20 \mathrm{mM}$ Tris $-\mathrm{HCl}(\mathrm{pH}$ 8.0) and $100 \mathrm{mM} \mathrm{NaCl}$. The phosphor storage plate was scanned, and the data for complex and free DNA bands were digitized for quantitative analysis. The [Protein $]_{50}$ values of PaSSB were determined using EMSA.

Table 3 The $[\text { Protein }]_{50}$ values of PaSSB mutants as analyzed by $\mathrm{EMSA}^{a}$

dT50

PaSSB

PaSSB(W40A)

$\operatorname{PaSSB}(\mathrm{W} 54 \mathrm{~A})$

PaSSB(F60A)

PaSSB(W88A)

${ }^{a}$ [Protein $]_{50}$ was calculated from the titration curves of EMSA by determining the concentration of the protein needed to achieve the midpoint value for input DNA binding. Errors are standard deviations determined by three independent titration experiments.

by ConSurf, ${ }^{37}$ revealing the degree of variability at each position along the primary sequence. The amino acid residues in the $\mathrm{C}$ terminal long glycine-rich hinge of PaSSB (aa 116-158) are variable (Fig. 3B). One PXXP motif (aa 125-PREP-128), known to mediate the protein-protein interactions, ${ }^{3}$ is found in the glycine-rich hinge of PaSSB.

\section{Crystal structure of PaSSB}

To understand the structure-function relationship of PaSSB, we crystallized PaSSB and determined its structure at a resolution of 2.04 $\AA$ (Table 2). Four monomers of PaSSB were found per asymmetric unit (Fig. 4A). Similar to EcSSB (Fig. 4B), the PaSSB monomer has an OB-fold domain, with the core bearing $\beta$ barrel capped by $\alpha$-helix. The entire C-terminal domain of PaSSB (aa 116-165) became disordered and disappeared. In the EcSSB-SSDNA complex, four essential aromatic residues, namely, Trp40, Trp54, Phe60, and Trp88, participated in ssDNA binding via stacking interactions. The corresponding residues in PaSSB are Trp40, Trp54, Phe60, and Trp88, which may play a similar role as that of EcSSB in ssDNA binding (Fig. 4C and D). Thus, the N-terminal domain of PaSSB is significantly similar to that of EcSSB in terms of sequence and structure.

\section{Mutational analysis}

According to crystal structure of PaSSB, we speculated that Trp40, Trp54, Phe60, and Trp88 in PaSSB allow nucleic acids to wrap around the whole PaSSB. We constructed and analyzed alanine substitution mutants through EMSA (Fig. 5). Table 3 summarizes [Protein $]_{50}$ of the binding of these PaSSB variants to dT50. These PaSSB mutants have [Protein $_{50}$ higher than that of the wild-type PaSSB. Thus, structure-based mutational analysis may indicate that PaSSB might bind to ssDNA in a manner similar to that of EcSSB.

\section{Conserved SSB binding site of PriA is present in PaPriA}

PriA interacts with the C-terminal acidic peptide of SSB at replication forks. This interaction is a driving force to stimulate the PriA activity. ${ }^{6}$ The crystal structure of KpPriA in complex with the acidic peptide of SSB (DDIPF) reveals a specific and conserved binding pocket in PriA. ${ }^{5}$ In the KpPriA-DDIPF complex, five residues, namely, Trp82, Tyr86, Lys370, Arg697, and Gln701, participate in SSB recognition. The corresponding residues in PaPriA are Trp87, Tyr91, Arg375, Arg704, and His708, which may play a similar role as that of KpPriA in KpSSB recognition. Arg697 in KpPriA is located near the $\alpha$-carboxyl group of the acidic peptide of SSB, and is known to play a critical role in altering the $\mathrm{SSB}_{35} / \mathrm{SSB}_{65}$ distribution. ${ }^{5}$ Such an important residue in PaPriA, namely, Arg704, is conserved (Fig. 6). Considering that PaPriA and KpPriA have a similar SSB-binding pocket and they may function similarly, we should assess whether the glycine-rich hinge that is not conserved between PaSSB and KpSSB is involved in the stimulation of the PriA helicase (see below).

\section{Protein chimeragenesis}

Given that EcPriA structure is not available (only KpPriA structure is present), we used KpPriA rather EcPriA in this study. To investigate which region in SSB is essential for stimulation of PriA, we used two chimeric proteins with different C-terminal domains (KpSSBn-PaSSBc and KpSSBn-StSSBc) for ATPase assay. As shown in Fig. 7, the KpPriA ATPase activity was dramatically stimulated (14-fold) by KpSSBn-StSSBc, but no effect was found when KpPriA interacted with KpSSBn-PaSSBc. Considering the identical N-terminal DNA-binding domain and the C-terminal acidic tail (DDDIPF) in both KpSSBn-PaSSBc and KpSSBn-StSSBc, we concluded that the flexible region at the Cterminus of SSB is the determinant for PriA stimulation specificity.

\section{The typical glycine-rich flexible region in PaSSB is not glycine- rich}

KpSSB, StSSB, and PaSSB share an overall 36\% sequence identity and are mostly conserved in the first 110 amino acids, which comprise the N-terminal ssDNA-binding domain. These SSBs also have identical C-terminal acidic tail sequences (Fig. 3A) used for binding partner proteins, such as PriA. ${ }^{5}$ KpSSB, StSSB, and KpSSBn-StSSBc could significantly stimulate KpPriA, but PaSSB and KpSSBn-PaSSBc could not. 
PaPriA MSDLILRLALPSPLRRLFDYRAPRGIPRSALQPGIRLLLPFGR-RELVGVLIEVTDRSEV KpPriA MS - VAHVALPVPLPRTFDYLLPEGM- - AVKAGCRVRVPFGK-QERIGIVAAVSERSEL EcPriA MP - - VAHVALPVPLPRTFDYLLPEGM - - TVKAGCRVRVPFGKQQERIGIVVSVSDASEL

PaPriA PEDKLKPARVLDAKPPMPAHLLELCRMTA OYYQH LGDTSWALPLLRQGEPEARQQ KpPriA PLDELKPVAEALDDEPVFSTTVWRLLMMAAEYYHHPIGDVLFHALPVMLRQGKPASATPL EcPriA PLNELKAVVEVLDSEPVFTHSVWRLLLMAADYYHHPIGDVLFHALPILLRQGRPAANAPM

PaPriA RFWHATAQSSLDDPR - LARAPRQRQALA I LKQHPHGVSHELLNQLE INKDSLDLLKEKGL KpPriA WYWFATEQGQVVDLNGLKRSRKQQQALAALRQGK - - I WRHQVGELEFNEAALQALRGKGL EcPriA WYWFATEQGQAVDLNSLKRSPKQQQALAALRQGK - - IWRDQVATLEFNDAALQALRKKGL

PaPriA VELEVRRHSTPPREGGW - - - L L LAELPLNPEQRAAFEAVRASHGGFHCFLLAGVTGSG KpPriA AELA- - C CEAPALDWRSAYSVAGERLRLNTEQATAVGA I HSAADRFSAWLLAGITGSG

EcPriA CDLA - . - SETPEFSDWRTNYAVSGERLRLNTEQATAVGA I HSAADTFSAWLLAGVTGSG

PaPriA KTEVYLQLIRETLAAGRQALVLIPEINLGPQTLARFERRFNARIALLHSALTDRERLDAW KpPriA KTEVYLSVLENVLAQGRQALVMVPEIGLTPQTIARFRQRFNAPVEVLHSGLNDSERLSAW EcPriA KTEVYLSVLENVLAQGKQALVMVPEIGLTPQTIARFRERFNAPVEVLHSGLNDSERLSAW

PaPriA LAARDGEADIVIGTRSALFTPLKRPGL I IVDEEHDASYKQQDGLRYHARDLALVRARLEN KpPriA LKAKNGEAA I VIGTRSSLFTPFKDLGVIVIDEEHDSSYKQQEGWRYHARDLAVWRAHSEQ EcPriA LKAKNGEAA I VIGTRSALFTPFKNLGVIVI DEEHDSSYKQQEGWRYHARDLAVYRAHSEQ

PaPriA VPILLGSATPALESLHNAQAGRYGLLRLTQRAGGAHPPKFIRLDVKSMPLDAGLSRPLQQ KpPriA IPI ILGSATPALETLHNVRQG|K|YRQLTLSKRAGNARPAQQHVLDLKGQPLQAGLSPALIS EcPriA IPI I LGSATPALETLCNVQQK KYRLLRLTRRAGNARPA I QHVLDLKGQKVQAGLAPAL I T

PaPriA AIGDTLAAGQQVLVFLNRRGFAPTLLCHDCGWISQCPRCDARMTVHQGSGELRCHHCDHR KpPriA RMRQHLQADNQVILFLNRRGFAPALLCHDCGWIAECPRCDSYYTLHQAQHHLRCHHCDSQ EcPriA RMRQHLQADNQVILFLNRRGFAPALLCHDCGWIAECPRCDHYYTLHQAQHHLRCHHCDSQ

PaPriA QRPPMNCPQCGKLDLRPLGAGTERAEERLRILFPNHPVLRIDRDSTSRKHAMRDLFATIN KpPriA RPIPRQCPSCGSTHLVPVGIGTEQLEQALAPLFPEVPISRIDRDTTSRKGALEEHLAAVH EcPriA RPVPRQCPSCGSTHLVPVGLGTEQLEQTLAPLFPGVPISRIDRDTTSRKGALEQQLAEVH

PaPriA SGEPCILVGTQMLAKGHHFPRVTLVAILDADGGLFSADFRASERMAQQIVQVAGRAGRAE KpPriA RGGARILIGTQMLAKGHHFPDVTLVSLLDVDGALFSADFRSAERFAQLYTQVSGRAGRAG EcPriA RGGARILIGTQMLAKGHHFPDVTLVALLDVDGALFSADFRSAERFAQLYTQAGRAGRAG

PaPriA EPGRVLIQTHLADHPLLVQLTEDGFAFAEQALSERRAAGLPPFAHLALLRAEAHKPGQA KpPriA KQGEVILQTHHPEHPLLQTLLYKGYDAFAEQALAERQTMQLPPWTSHVLIRAEDHNNQQA EcPriA KQGEVVLQTHHPEHPLLQTLLYKGYDAFAEQALAERRMMQLPPWTSHVIVRAEDHNNQHA

PaPriA EAFLDSACSAAEQLLEQMGgPEVELLGPVPAPMERRAGKHRAQLLLQCMSRAPL HRLTP 713 KpPriA PLFLQQLRNLL - - QASPLADEKLWVLGPVPALAPKRGGRWRWQILLQHPSRVRL|HIVSG 706 EcPriA PLFLQQLRNLI - - LSSPLADEKLWVLGPVPALAPKRGGRWRWQ I LLQHPSEVLQH I ING 707

PaPriA WLQSLEQLPGGRQVRWSLDIDPIDLF 739

KpPriA TLALINTLPEARKVKWVLDVDPIEG-

EcPriA TLALINTIPDSRKVKWVLDVDPIEG- 732

Fig. 6 Multiple amino acid sequence alignment of PriA proteins. Sequence alignment of PaPriA, KpPriA, and EcPriA was generated by CLUSTALW2. Identical amino acid residues are colored in red. Amino acid residues with similar properties judged by CLUSTALW2 are colored in light blue. In the KpPriA-DDIPF complex, Trp82, Tyr86, Lys370, Arg697, and Gln701 participate in SSB recognition. The corresponding residues in PaPriA are Trp87, Tyr91, Arg375, Arg704, and His708 (boxed), which may play a similar role as that of KpPriA in KpSSB recognition.

Although PaSSB has the PXXP motif at the C-terminus (aa 125128), PaSSB was found to be incapable of stimulating PaPriA. PaSSB is similar to EcSSB in terms of sequence and structure (Fig. 4), except for the presence of a long glycine-rich flexible region. PaSSB (aa 116-165) contains only one Gly residue, which is significantly less than those in KpSSB (aa 116-174, with 11 Gly residues) and StSSB (aa 117-176, with 12 Gly residues). Thus, the glycine-rich flexible region in PaSSB may not be very flexible, and the region in PaSSB is indeed not glycine-rich (Fig. 3). 
A

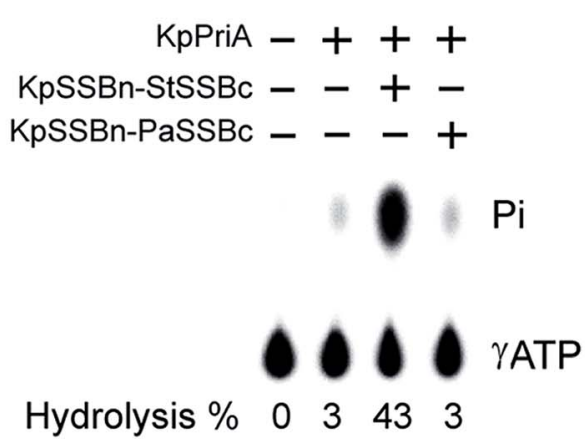

B

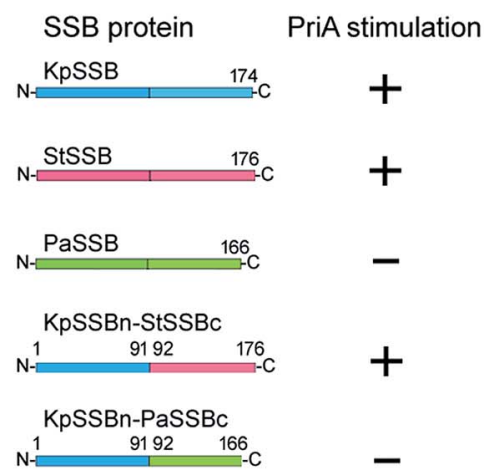

Fig. 7 (A) KpSSBn-StSSBc can stimulate KpPriA activity, but KpSSBn-PaSSBc cannot. KpPriA ATPase assay was performed with KpSSBn-StSSBc $(10 \mu \mathrm{M})$ or KpSSBn-PaSSBc $(10 \mu \mathrm{M})$. (B) On the basis of results in this study, the glycine-rich flexible region among SSB species is a determinant of PriA stimulation.

Table 4 The PXXP motifs $^{a}$

\begin{tabular}{lllll}
\hline & EcSSB & KPSSB & StSSB & PaSSB \\
\hline First motif & PQQP & PQQP & PQQPQQP & PREP \\
Second motif & PQQS & PQQQ & PQQS & PAPQQ \\
Third motif & PAAP & PAAP & PAP & PAPQP
\end{tabular}

${ }^{a}$ In EcSSB, the PXXP motifs occur at residues 139, 156 and 161 Correspondingly, the putative PXXP motifs in KpSSB, StSSB and PaSSB are shown.

\section{The PXXP motifs}

The PXXP motifs in the glycine-rich hinge of EcSSB are known to mediate the protein-protein interactions. ${ }^{38}$ In EcSSB, the PXXP motifs occur at residues 139 (PQQP), 156 (PQQS) and 161 (PAAP). ${ }^{3}$ The corresponding motifs in KpSSB are PQQP, PQQQ, and PAAP, respectively. In StSSB, the first motif is duplicated to PQQPQQP while the third motif is shortened to PAP instead of PAAP in EcSSB. Given that PriA activity could be stimulated by KpSSB and StSSB (Fig. 2), these pseudo-PXXP motifs in KpSSB and StSSB may function well as those in EcSSB. In PaSSB, the second and third PXXP motifs are not significant. The first PXXP motif is PREP. The putative second and third PXXP motifs are PAPQQ and PAPQP, respectively. These motifs are instead PXPXX, being interrupted or lengthened by the additional residue (Table 4). This may be the reason why PaSSB could not enhance the activity of PriA. However, the cause could be the linker the motifs or both. At this time, it remains unclear whether the PXXP motifs established from EcSSB study are also applicable to other SSBs.

\section{Both the SSB-Ct and the glycine-rich flexible region in SSB is crucial for PriA stimulation}

Recently, we have found that $\mathrm{SsbA},{ }^{29} \mathrm{SsbB}^{28}$ and SsbC, ${ }^{19}$ cannot stimulate PriA activity. Unlike $E$. coli, the C-terminus in these Gram-positive bacterial SSBs are different. PaSSB has an acidic peptide at the C-terminus like EcSSB, but it was still incapable of stimulating PriA activity (Fig. 2). The length of the glycinerich hinge in EcSSB is known to affect EcPriA activity. ${ }^{24}$ The glycine-rich flexible region in PaSSB is 12 residues shorter than that in EcSSB (Fig. 3). The glycine-rich flexible region of PaSSB is quite different from that of other SSBs in terms of the length and the amino acid sequence (Fig. 3). Given that this flexible region in SSB is crucial for protein binding, ${ }^{3}$ this may be a reason for the different effects of SSB on PriA stimulation.

In conclusion, protein chimeragenesis and structure-function analyses indicate that the inherent difference in the glycine-rich flexible region among SSB species is a determinant of PriA stimulation. Further research can directly focus on determining how the glycine-rich hinge of SSB activates PriA activity and the reason why bacterial SSBs need to evolve different C-terminal domains during evolution.

\section{Conflicts of interest}

There are no conflicts of interest to declare.

\section{Acknowledgements}

We would like to thank two anonymous reviewers and the editor for their comments. We thank the experimental facility and the technical services provided by the Synchrotron Radiation Protein Crystallography Facility of the National Core Facility Program for Biotechnology, Ministry of Science and Technology and the National Synchrotron Radiation Research Center, a national user facility supported by the Ministry of Science and Technology, Taiwan, ROC. This research was supported by a grant from the Ministry of Science and Technology, Taiwan (MOST 107-2320-B-040-014 to C. Y. Huang).

\section{References}

1 R. R. Meyer and P. S. Laine, Microbiol. Rev., 1990, 54, 342380.

2 S. Raghunathan, A. G. Kozlov, T. M. Lohman and G. Waksman, Nat. Struct. Biol., 2000, 7, 648-652.

3 P. R. Bianco, Prog. Biophys. Mol. Biol., 2017, 127, 111-118.

4 E. Antony and T. M. Lohman, Semin. Cell Dev. Biol., 2018, DOI: 10.1016/j.semcdb.2018.03.017. 
5 B. Bhattacharyya, N. P. George, T. M. Thurmes, R. Zhou, N. Jani, S. R. Wessel, S. J. Sandler, T. Ha and J. L. Keck, Proc. Natl. Acad. Sci. U. S. A., 2014, 111, 1373-1378.

6 C. J. Cadman and P. McGlynn, Nucleic Acids Res., 2004, 32, 6378-6387.

7 T. A. Windgassen, S. R. Wessel, B. Bhattacharyya and J. L. Keck, Nucleic Acids Res., 2018, 46, 504-519.

8 Y. H. Huang and C. Y. Huang, BioMed Res. Int., 2014, 2014, 195162.

9 R. C. Heller and K. J. Marians, Nat. Rev. Mol. Cell Biol., 2006, 7, 932-943.

10 C. C. Huang and C. Y. Huang, Biochem. Biophys. Res. Commun., 2016, 477, 988-992.

11 Y. H. Huang and C. Y. Huang, Biochem. Biophys. Res. Commun., 2013, 442, 147-152.

12 T. Aramaki, Y. Abe, T. Ohkuri, T. Mishima, S. Yamashita, T. Katayama and T. Ueda, Genes Cells, 2013, 18, 723-732.

13 T. Aramaki, Y. Abe, K. Furutani, T. Katayama and T. Ueda, J. Biochem., 2015, 157, 529-537.

14 S. Fujiyama, Y. Abe, J. Tani, M. Urabe, K. Sato, T. Aramaki, T. Katayama and T. Ueda, FEBS J., 2014, 281, 5356-5370.

15 M. Lopper, R. Boonsombat, S. J. Sandler and J. L. Keck, Mol. Cell, 2007, 26, 781-793.

16 K. Bush, Curr. Opin. Microbiol., 2010, 13, 558-564.

17 W. H. Zhao and Z. Q. Hu, Crit. Rev. Microbiol., 2010, 36, 245258.

18 J. G. Glanzer, J. L. Endres, B. M. Byrne, S. Liu, K. W. Bayles and G. G. Oakley, J. Antimicrob. Chemother., 2016, 71, 34323440.

19 Y. H. Huang and C. Y. Huang, Oncotarget, 2018, 9, 2023920254.

20 Y. H. Huang, Y. H. Lo, W. Huang and C. Y. Huang, Genes Cells, 2012, 17, 837-849.

21 Y. H. Huang, Y. L. Lee and C. Y. Huang, Protein J., 2011, 30, 102-108.

22 H. C. Jan, Y. L. Lee and C. Y. Huang, Protein J., 2011, 30, 2026.

23 Y. H. Huang and C. Y. Huang, BioMed Res. Int., 2014, 2014, 573936.
24 H. Y. Tan, L. A. Wilczek, S. Pottinger, M. Manosas, C. Yu, T. Nguyenduc and P. R. Bianco, Protein Sci., 2017, 26, 700717.

25 C. Y. Huang, C. H. Hsu, Y. J. Sun, H. N. Wu and C. D. Hsiao, Nucleic Acids Res., 2006, 34, 3878-3886.

26 J. H. Liu, T. W. Chang, C. Y. Huang, S. U. Chen, H. N. Wu, M. C. Chang and C. D. Hsiao, J. Biol. Chem., 2004, 279, 50465-50471.

27 Y. H. Huang, Y. H. Lo, W. Huang and C. Y. Huang, Genes Cells, 2012, 17, 837-849.

28 K. L. Chen, J. H. Cheng, C. Y. Lin, Y. H. Huang and C. Y. Huang, RSC Adv., 2018, 8, 28367-28375.

29 Y. H. Huang, H. H. Guan, C. J. Chen and C. Y. Huang, PLoS One, 2017, 12, e0182060.

30 Y. H. Huang, Y. Lien, C. C. Huang and C. Y. Huang, PLoS One, 2016, 11, e0157593.

31 Z. Otwinowski and W. Minor, Methods Enzymol., 1997, 276, 307-326.

32 A. J. McCoy, R. W. Grosse-Kunstleve, P. D. Adams, M. D. Winn, L. C. Storoni and R. J. Read, J. Appl. Crystallogr., 2007, 40, 658-674.

33 J. J. Headd, N. Echols, P. V. Afonine, R. W. Grosse-Kunstleve, V. B. Chen, N. W. Moriarty, D. C. Richardson, J. S. Richardson and P. D. Adams, Acta Crystallogr., Sect. D: Biol. Crystallogr., 2012, 68, 381-390.

34 P. Emsley and K. Cowtan, Acta Crystallogr., Sect. D: Biol. Crystallogr., 2004, 60, 2126-2132.

35 C. Y. Huang, Determination of the binding site-size of the protein-DNA complex by use of the electrophoretic mobility shift assay, in Stoichiometry and Research: The Importance of Quantity in Biomedicine, ed. A. Innocenti, InTech Press. Rijeka, Croatia, 2012, pp.235-242.

36 T. M. Lohman, E. J. Tomko and C. G. Wu, Nat. Rev. Mol. Cell Biol., 2008, 9, 391-401.

37 M. Landau, I. Mayrose, Y. Rosenberg, F. Glaser, E. Martz, T. Pupko and N. Ben-Tal, Nucleic Acids Res., 2005, 33, W299-W302.

38 P. R. Bianco, S. Pottinger, H. Y. Tan, T. Nguyenduc, K. Rex and U. Varshney, Protein Sci., 2017, 26, 227-241. 